OPEN ACCESS

Edited by: John Austin Vargo,

University of Pittsburgh Medical

Center, United States

Reviewed by:

Joshua L. Rodríguez-López,

University of Pittsburgh Medical

Center, United States

Joshua Silverman,

New York University, United States

Scott Glaser,

City of Hope National Medical Center,

United States

*Correspondence:

Brian T. Collins

collinsb@gunet.georgetown.edu

Specialty section:

This article was submitted to

Radiation Oncology,

a section of the journal

Frontiers in Oncology

Received: 14 June 2020 Accepted: 23 September 2020 Published: 10 December 2020

Citation:

Aghdam N, Repka MC, McGunigal M,

Pepin A, Paydar I, Rudra S, Paudel N,

Pernia Marin M, Suy S, Collins SP,

Barnes W and Collins BT (2020)

Stereotactic Body Radiation Therapy:

A Versatile, Well-Tolerated, and

Effective Treatment Option for

Extracranial Metastases From Primary

Ovarian and Uterine Cancer.

Front. Oncol. 10:572564.

doi: 10.3389/fonc.2020.572564

\section{Stereotactic Body Radiation Therapy: A Versatile, Well-Tolerated, and Effective Treatment Option for Extracranial Metastases From Primary Ovarian and Uterine Cancer}

\author{
Nima Aghdam ${ }^{1,2}$, Michael C. Repka ${ }^{3}$, Mary McGunigal ${ }^{1}$, Abby Pepin ${ }^{4}$, Ima Paydar ${ }^{5}$, \\ Sonali Rudra ${ }^{1}$, Nitika Paudel ${ }^{1}$, Monica Pernia Marin ${ }^{6}$, Simeng Suy ${ }^{1}$, Sean P. Collins ${ }^{1}$, \\ Willard Barnes ${ }^{7}$ and Brian T. Collins ${ }^{1 *}$

\begin{abstract}
Department of Radiation Medicine, Georgetown University Hospital, Washington, DC, United States, ${ }^{2}$ Department of Radiation Oncology, Beth Israel Deaconess Medical Center and Harvard Medical School, Boston, MA, United States, ${ }^{3}$ Department of Radiation Oncology, New York University Winthrop Hospital, Mineola, NY, United States, ${ }^{4}$ School of Medicine and Health Sciences, George Washington University, Washington, DC, United States, ${ }^{5}$ Department of Radiation Oncology, University of Pennsylvania, Philadelphia, PA, United States, ${ }^{6}$ Geriatric and Palliative Medicine Division, George Washington University Hospital, Washington, DC, United States, ${ }^{7}$ Division of Gynecologic Oncology, Georgetown University Hospital, Washington, DC, United States
\end{abstract}

Purpose: Single extracranial metastases from ovarian and uterine malignancies have historically been treated with surgery or conventional radiation. We report mature local control (LC), overall survival (OS), progression free survival (PFS), and toxicity for patients who completed 5 -fraction stereotactic body radiation therapy (SBRT).

Methods: Patients with biopsy-proven, single extracranial metastases from primary ovarian and uterine malignancies treated with 5 -fraction SBRT were included. Patients were stratified based on tumor volume (small $<50 \mathrm{cc}$ or large $\geq 50 \mathrm{cc}$ ) and dose (low dose $<35$ Gy or high $\geq 35$ Gy). Kaplan-Meier method was used to estimate LC, OS, and PFS.

Results: Between July 2007 and July 2012, 20 patients underwent SBRT to a single extracranial metastasis. Primary site was divided evenly between ovarian and uterine $(\mathrm{n}=$ 10 each). Metastases involved the liver (30\%), abdominal lymph nodes (25\%), lung (20\%), pelvic lymph nodes (10\%), spine (10\%), and extremity (5\%). The median gross tumor volume (GTV) was $42.5 \mathrm{cc}$ (range, 5-273 cc) and the median dose to the GTV was 35 Gy (range, 30-50 Gy). At a median follow-up of 56 months, the 5-year LC and OS estimates were 73 and $46 \%$. When stratified by tumor volume, the 5 -year LC and OS for small tumors were significantly better at 100\% $(p<0.01)$ and 65\% $(p<0.02)$. When stratified by dose, the 5 -year LC was $87.5 \%$ with high dose and $53.6 \%$ with low dose $(p=0.035)$. The 5 -year PFS for the entire cohort was $20 \%$. Four patients with small metastases who had complete response remained disease free at study completion and were considered cured (median PFS > 10 years). Treatment was generally well tolerated, and only one patient experienced a late grade III musculoskeletal SBRT related toxicity. 
Conclusions: SBRT is a versatile, well-tolerated, and effective treatment option for single extracranial metastases from ovarian and uterine primary tumors. 35 Gy in five fractions appears to be a practical minimum effective dose. Four patients with small metastases were disease free at the study completion and considered cured. However, patients with larger metastases $(\geq 50 \mathrm{cc}$ ) may require higher SBRT dosing or alternative treatments.

Keywords: radiation, stereotactic body radiation therapy, ovarian cancer, uterine cancer, metastatic, oligometastatic

\section{INTRODUCTION}

Ovarian and uterine cancer remain the fifth $(13,980)$ and sixth $(12,160)$ leading causes of cancer related death in U.S. women despite improvement in therapy (1). Systemic therapies form the cornerstone of treatment for metastatic tumors. However, aggressive local therapy in a selected patient population with an eradicated primary tumor and limited metastatic disease $(\leq 5$ metastases), has yielded promising results $(2,3)$. For these oligometastatic patients, metastasectomy can provide lengthy disease-free intervals in conjunction with standard systemic therapies (4).

Not all patients are surgical candidates, either due to their comorbidities or unfavorable sites of metastases. In such cases, ablative radiation therapy may prove to be an ideal local therapy. The outcomes of conventional radiation in the treatment of metastatic disease has been disappointing due to significant local failure rates, particularly for tumors with unfavorable histology such as sarcomas $(5,6)$. Furthermore, the delivery of the high doses necessary to eradicate gross disease in the lung, liver, and peritoneal cavity leads to unacceptable toxicity with conventional techniques.

Historically, low dose (30 Gy in 20 fractions) whole abdominal irradiation (WAI) was utilized to treat predominately radiosensitive ovarian and uterine tumors following gross total resection with good long-term local control of microscopic peritoneal disease (7-9). Adjuvant chemotherapy has now emerged as the preferred treatment for advanced ovarian and uterine malignancies following radical surgery. In the oligometastatic setting however, in order to achieve long-term disease-free survival it may be necessary to eradicate sites of chemotherapy resistant gross disease with treatments other than surgery (10). Fortunately, over the past two decades, stereotactic body radiation therapy (SBRT) has emerged as the preferred method to deliver ablative radiation doses to a wide range of extracranial tumors with exceptional precision and safety (4). Visceral metastases involving both the lung and liver have been successfully eradicated with SBRT and such treatment has prolonged survival in select groups of patients $(11,12)$. Further refinement to this technique has come in the form of real-time tumor tracking technologies which may enhance the safety profile by reducing the treatment margins mandatory for achieving durable local control $(13,14)$.

The aim of this study was to treat a selected subset of oligometastatic ovarian and uterine cancer patients with minimal disease, identify clinical features that could predict long-term disease-free survival, and establish a safe minimum effective dose in five fractions for this cohort. Here we report the mature results from 20 consecutive patients with single extracranial metastases arising from previously eradicated ovarian and uterine primary tumors treated with 5-fraction robotic SBRT.

\section{MATERIALS AND METHODS}

\section{Patient Selection}

The Georgetown University Institutional Review Board (IRB) approved this single institution retrospective review. Consecutive patients treated per an institutional protocol who had single extracranial metastases and a controlled ovarian or uterine primary tumor were eligible for this study. Biopsy confirmation of the metastatic site and concordance with the patient's original pathologic diagnosis were required for study inclusion. Vaginal cuff failures, multiple sites of metastases and re-irradiation were considered exclusion criteria. A single metastasis was defined as the only gross disease present following the last course of therapy. Extent and frequency of prior cytoreductive procedures or systemic agents were not exclusion criteria. Baseline PET/CT with IV contrast was performed for each patient when feasible. Eastern Cooperative Oncology Group performance status of two or less was required for inclusion. Patients were stratified based on tumor volume (small tumors $<50 \mathrm{cc}$ or large tumors $\geq 50 \mathrm{cc}$ ) and dose (low dose $<35$ Gy or high dose $\geq 35$ Gy). The decision to proceed with SBRT in lieu of surgery was reached in consultation with the gynecologic oncologist (WB). All patients included in this analysis were treated with robotic SBRT in five fractions using the CyberKnife Robotic Radiosurgery System (Accuray Inc., Sunnyvale, CA, USA).

\section{Fiducial Placement}

Tumor tracking based on translational and rotational target information routinely requires the use of a minimum of three non-colinear fiducials to be visible on the orthogonal images of the CyberKnife $\mathrm{x}$-ray targeting system. Therefore, an interventional radiologist or pulmonologist inserted three to five gold fiducials via CT guidance or bronchoscopy in or near tumors susceptible to intrafraction motion (13). An exception to this practice is spine or paraspinal tumors, which were treated using spine tracking (14). 


\section{Treatment Planning and Delivery}

Patients were simulated in the supine treatment position with arms at sides using high resolution (1.25 mm slices) contrast enhanced planning CT. Gross tumor volumes (GTVs) as well as organs at risk (OARs) of developing radiation damage were contoured by the treating radiation oncologist $(\mathrm{BC})$. For stationary tumors, the GTV was the planning target volume (PTV). However, for lung and liver tumors that move with respiration, the GTV margin was uniformly expanded by $5 \mathrm{~mm}$ to establish the PTV margin. Clinical target volumes (CTVs), generally used to account for microscopic disease spread, were not used in this study. A treatment plan was generated using the MultiPlan (Accuray Inc., Sunnyvale, CA, USA) nonisocentric, inverse-planning algorithm with tissue density heterogeneity corrections for lung based on an effective depth correction (13). Treatment plans included more than 100 nonisocentric pencil beams. The radiation dose was divided into five equal fractions, prescribed to an isodose line that covered at least 95\% of the PTV. Dose constraints for OARs were consistent with the widely accepted AAPM TG-101 report (15). Patients were treated per previously published detailed institutional protocols $(13,14)$. A representative treatment plan and tumor response are illustrated in Figure 1.

\section{Follow-Up}

Patients were followed per routine institutional practice. Local failure was defined as progression of disease on CT or PET imaging as determined by the treating radiation oncologist (BC) and gynecologic oncologist (WB). Equivocal findings were dated as time of failure upon confirmation of disease progression at the next follow up imaging. Toxicities were scored according to the National Cancer Institute Common Terminology Criteria for Adverse Events, Version 4.0 (16).

\section{Statistical Analyses}

All outcomes were calculated from the date of completion of SBRT treatment to the last date of known follow-up or death. Actuarial local control (LC), overall survival (OS), and progression free survival (PFS) were calculated and compared using the Kaplan-Meier method and log-rank test. Statistical analysis was performed using SPSS Statistics version 23.0 (IBM Corporation, Armonk, NY, USA).

\section{RESULTS}

\section{Overview}

Between July 2007 and July 2012, a total of 20 patients were identified meeting study inclusion criteria. The median patient age was 64 years (range, 47-92) and the median ECOG performance status score was 0 (range, 0-1). Ninety-five percent of patients had completed chemotherapy at some point in their clinical course prior to SBRT. The median GTV was 42.5 cc (range, 5.0-273.0). The median prescription dose was 35 Gy (range, 30-50), with a median daily dose of 7 Gy (range, 6-10). All patients were treated in five fractions over a median 7-day period (range, 5-9). The median prescription isodose line was $76 \%$ (range, $70-84 \%$ ), with a median maximum plan point dose of 43.7 Gy (range, 37.1-61.3 Gy). The median cumulative prescription equivalent dose in 2 Gy fractions (EQD2) was 50 Gy $(\alpha / \beta=10)$. Patient and treatment characteristics are presented in Table 1.

Primary tumor site was divided evenly between ovarian and uterine $(\mathrm{n}=10 \mathrm{each})$. The most common histology was endometrioid adenocarcinoma ( $\mathrm{n}=6,30 \%$ ), followed by papillary serous carcinoma $(\mathrm{n}=4,20 \%)$. Other tumors included granulosa cell tumor $(n=2)$, carcinosarcoma $(n=2)$, clear cell carcinoma $(n=2)$, leiomyosarcoma $(n=2)$, and one mucinous adenocarcinoma and undifferentiated carcinoma each. Eleven metastases involved the abdomen, nearly evenly distributed between the liver $(n=6)$ and para-aortic lymph nodes $(n=5)$. Four metastases were located in the lung, two were located within pelvic lymph nodes, two were located within the spine and one was located in the thigh. Individual tumor characteristics are summarized in Table 2.

TABLE 1 | Baseline patient and treatment characteristics.

\begin{tabular}{ll} 
Characteristic & Median (Range) \\
\hline Age (years) & $64(44-92)$ \\
ECOG PS (score) & $0(0-1)$ \\
Gross Tumor Volume (cc) & $42.5(5.0-273.0)$ \\
Prescription IDL (\%) & $76.0(70.0-84.0)$ \\
Prescription Dose (Gy) & $35.0(30.0-50.0)$
\end{tabular}

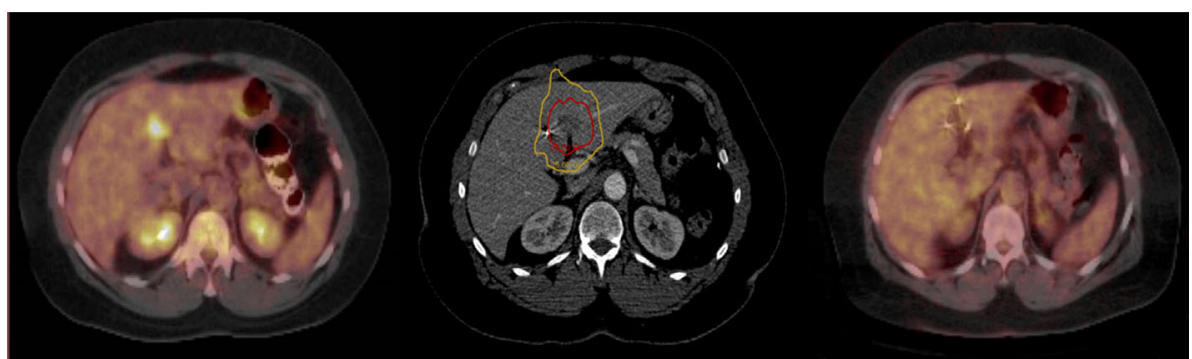

FIGURE 1 | Pre-treatment PET/CT with a single liver metastasis (Left); Red prescription isodose line encompassing the liver metastasis (Center); PET/CT at 6 months post-treatment demonstrating eradication of the liver metastasis (Right). 


\section{Local Control, Overall Survival, and Progression Free Survival}

At a median follow-up of 56 months, there were four local failures. Local control rates at 2 and 5 years were 82 and $73 \%$ (Figure 2A). When stratified by size, the LC at 5 years was $100 \%$ in patients with tumors $<50 \mathrm{cc}$ and $0 \%$ in larger tumors $(\mathrm{p}<$ 0.01 ) (Figure 2B). Only one patient treated with high-dose SBRT $(n=12)$ experienced a local failure compared to three patients treated with low-dose SBRT ( $n=8, p=0.035)$ (Figure 2C). The four local failures occurred in a para-aortic lymph node, pelvic lymph node, liver, and thigh.

Median OS was 4 years. Two-year and 5-year OS where 65 and $46 \%$ (Figure 2D). When stratified by size OS at 5 years was $65 \%$ for smaller tumors and $25 \%$ for larger tumors $(p<0.02)$

(Figure 2E). Median PFS was 9 months. Forty-five percent of patients remained disease free at 1 year with the rates decreasing to 30 and $20 \%$ at 2 and 5 years (Figure 2F). All deaths were attributed to metastatic disease. At the time of the writing of this report four patients with biopsy proven metastases remain disease free and were considered cured at a median overall survival of 126 months (range, 68-151 months) following SBRT alone. These tumors were relatively small (median size $20 \mathrm{cc}$ ), involved favorable locations including the lung (2), liver and paraaortic lymph node and received a relatively high mean dose of 40 Gy (range, 35-45). Three of the cured tumors where relatively indolent endometrioid adenocarcinoma metastases, and one was a potentially more aggressive liver ovarian papillary serous carcinoma metastasis in a BRCA mutation free patient (Table 2).

\section{Toxicity}

Overall, the treatment was well tolerated with no acute grade III or higher toxicity and only one reported late grade III toxicity. The most common acute toxicities were fatigue $(n=5)$ and nausea $(n=5)$. Nausea requiring medication occurred in three patients with abdominal lesions $(\mathrm{n}=3)$. One late lumbar vertebral body compression fracture was observed approximately 7 years following the treatment of a para-aortic lymph node metastasis (30 Gy) in a patient with osteopenia.

\section{DISCUSSION}

In the earliest gynecological oligometastatic SBRT prospective trial of its kind, Kunos et al. treated 50 patients with a wide variety of primary tumors between July 2009 and September 2011 (17). The majority were ovarian adenocarcinoma (50\%) or uterine adenocarcionoma (28\%) with the remainder being either squamous cell carcinoma of the cervix (18\%) or vulva (4\%). Only $28 \%$ of the patients had single metastases. Most patients had two or three metastases (68\%) involving lymph nodes (68\%). However, metastases involving the liver (16\%), lung (8\%), and spine (4\%) were also treated. Prior chemotherapy (94\%), adjuvant chemotherapy (8\%), and re-irradiation (32\%) were permitted. Appropriately, for such a diverse group of patients and targets being treated on a Phase II clinical trial, a relatively low dose of $24 \mathrm{~Gy}$ in three fractions (prescription EQD2 = $36 \mathrm{~Gy}$ with $\alpha / \beta=10$ ) was safely delivered. Surprisingly, no SBRT targeted disease progressed during the fairly brief 15 -month median follow-up period for surviving patients (range, 1-31 months). Nonetheless, median PFS and OS were only 7.8 and 20.2 months prompting the investigators to explore combined modality approaches for this patient population.

Recently, Lazzari et al. reported their oligometastatic ovarian cancer SBRT outcomes (18). Eighty two patients with 156 metastases were treated between May 2012 and December 2016. A sizable majority of patients had single metastases (70.7\%) involving lymph nodes (72\%). However, metastases involving the

TABLE 2 | Individual tumor characteristics.

\begin{tabular}{|c|c|c|c|c|c|}
\hline Patient & Organ & Histology & Site & GTV (cc) & Dose (Gy) \\
\hline 1 & Ovary & Granulosa Cell Tumor & Spine & 10 & 30 \\
\hline $2^{\dagger}$ & Uterus & Endometriod Adenocarcinoma & Lung & 20 & 45 \\
\hline $3^{\dagger}$ & Uterus & Endometriod Adenocarcinoma & Abdomen & 5 & 35 \\
\hline $4^{\dagger}$ & Ovary & Papillary Serous Carcinoma & Liver & 20 & 45 \\
\hline 5 & Uterus & Endometriod Adenocarcinoma & Spine & 96 & 35 \\
\hline 6 & Uterus & Carcinosarcoma & Abdomen & 51 & 30 \\
\hline 7 & Uterus & Carcinosarcoma & Abdomen & 30 & 30 \\
\hline 8 & Ovary & Clear Cell Carcinoma & Lung & 46 & 50 \\
\hline 9 & Ovary & Undifferentiated Carcinoma & Liver & 22 & 50 \\
\hline 10 & Ovary & Papillary Serous Carcinoma & Liver & 97 & 35 \\
\hline 11 & Ovary & Granulosa Cell Tumor & Abdomen & 39 & 30 \\
\hline 12 & Ovary & Mucinous Adenocarcinoma & Liver & 24 & 35 \\
\hline 13 & Ovary & Papillary Serous Carcinoma & Liver & 108 & 30 \\
\hline 14 & Uterus & Endometriod Adenocarcinoma & Abdomen & 36 & 35 \\
\hline 15 & Uterus & Leiomyosarcoma & Thigh & 273 & 40 \\
\hline 16 & Uterus & Leiomyosarcoma & Pelvis & 53 & 30 \\
\hline 17 & Uterus & Endometriod Adenocarcinoma & Lung & 63 & 40 \\
\hline $18^{\dagger}$ & Uterus & Endometroid Adenocarcinoma & Lung & 48 & 40 \\
\hline 19 & Ovary & Clear Cell Carcinoma & Pelvis & 166 & 30 \\
\hline 20 & Ovary & Papillary Serous Carcinoma & Liver & 28 & 30 \\
\hline
\end{tabular}

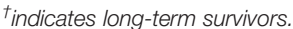




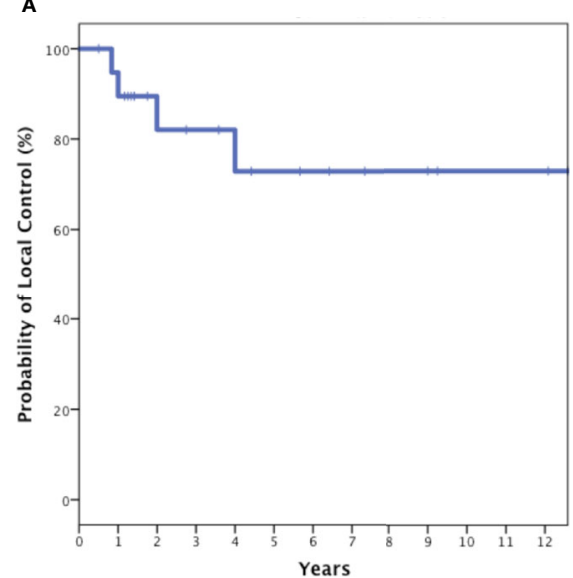

c

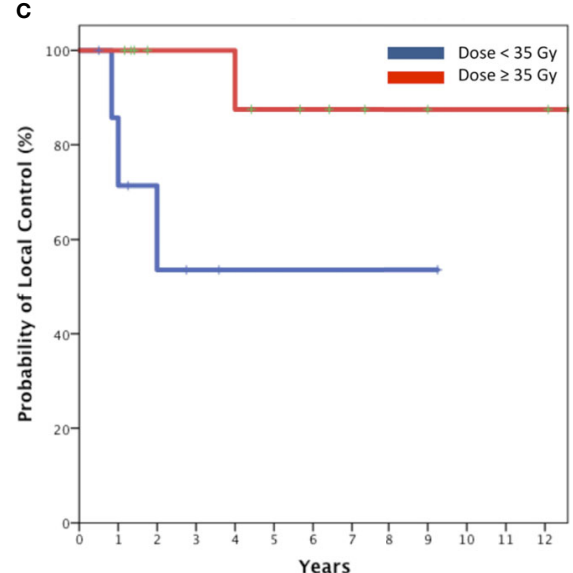

E

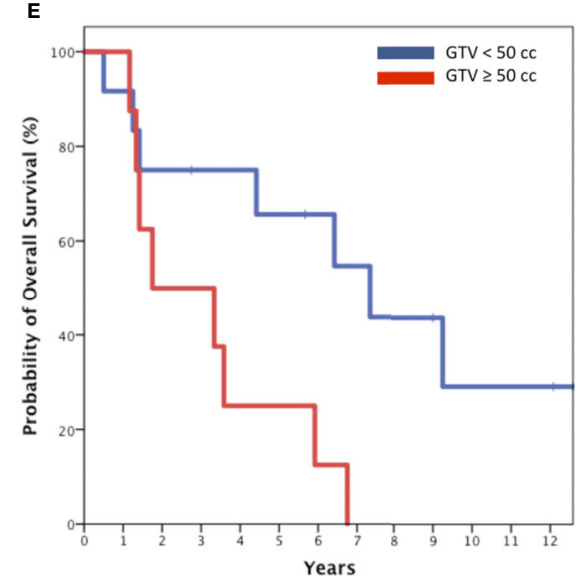

B

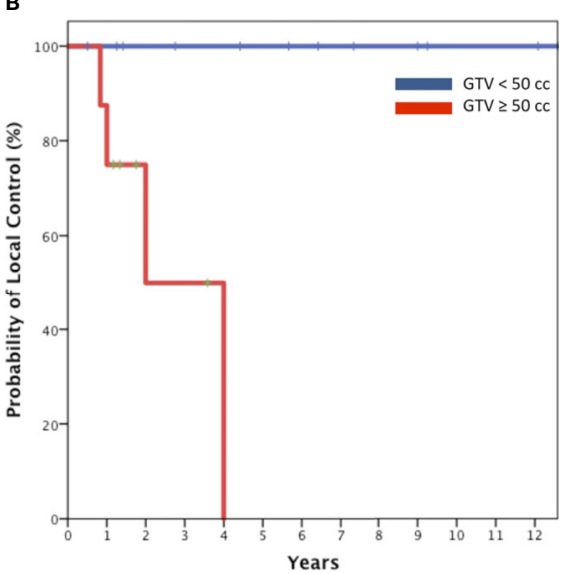

D

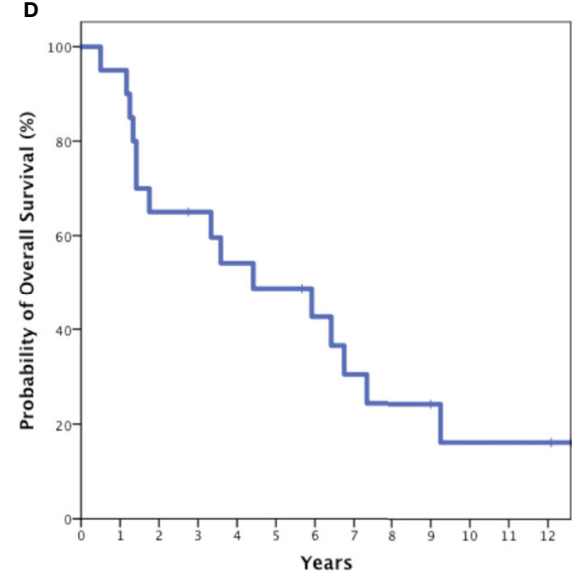

$\mathbf{F}$

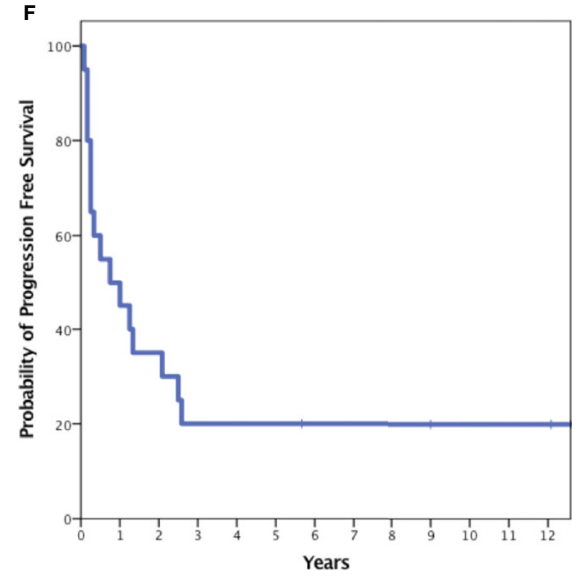

FIGURE 2 | Kaplan-Meier plot of Local control for the entire cohort (A); local control stratified by size (B) and dose (C); Overall survival for the entire cohort and (D) and stratified by size (E); progression free survival for entire cohort (F).

liver (9\%) and lung (4\%) were also treated. Metastases were generally small with a median GTV of 6.77 cc (range, 0.19-90.50 cc). Prior chemotherapy (98.8\%), concomitant chemotherapy (18.6\%), and re-irradiation (3.2\%) were permitted. A relatively low median prescription dose of 24 Gy (range, 14-45 Gy) was generally delivered in three fractions (range, 1-5 fractions) with limited acute toxicity. At a median follow-up of 17.4 months, the actuarial 2-year local progression-free survival was $68 \%$. However, OS at 2 years was $71 \%$, likely resulting from the high number of patients with small single lymph node metastases. Nonetheless, the median systemic treatment-free interval after SBRT was 7.4 months, mirroring the Kunos et al. experience. 
Our series differs from contemporaneous SBRT series in that all patients were required to have a single metastasis, and reirradiation was not permited $(17,18)$. The single metastasis requirement was necessary in order to lengthen our cohorts' OS allowing for the testing of our proposed hypotheses. The exclusion of re-irradiation patients was necessary so as not to interfere with our planned minimum effective SBRT dose analysis. Consistent with contemporaneous SBRT studies, the majority of our patients completed chemotherapy prior to SBRT (95\%). Over a 5-year period extending from July 2007 to July 2012, we treated 20 patients with single biopsy proven ovarian (50\%) and uterine (50\%) cancer oligometastases. Half of the metastases involved mobile visceral organs including the liver (30\%) and lung (20\%). The other half involved static lymph nodes $(35 \%)$ or musculoskeletal structures $(15 \%)$. At a median follow-up of 56 months, our 2-year and 5-year OS were 65 and $46 \%$, justifying our selection criteria.

First, we hypothesized that a potentially curable oligometastatic state exists in patients with single extracranial ovarian and uterine cancer metastases and that these patients are potentially curable with SBRT alone (19). At a median follow-up of 56 months (range, 6-151 months), the 5-year LC and OS estimates were a reasonable 73 and $46 \%$. However, when stratified by GTV, the 5-year LC and OS for small tumors $(<50 \mathrm{cc})$ were significantly better at $100 \%(\mathrm{p}<$ $0.01)$ and $65 \%(p<0.02)$. Furthermore, at the time of the writing of this report, four biopsy proven oligometastatic patients (20\% of the study cohort) remain alive and disease free at a median follow-up of greater than 10 years without post-treatment systemic therapy further bolstering our hypothesis. These four patient's metastases were relatively small with a median volume of $20 \mathrm{cc}$ (range, 5-48 cc), of fairly indolent histology (75\% endometroid adenocarcinoma/25\% papillary serous carcinoma) and involved favorable locations for high-dose SBRT delivery (median dose 40 Gy) as illustrated in Table 2. Our research suggests that future studies should at a minimum evaluate the role of SBRT in the treatment of small endometroid adenocarcinoma and ovarian papillary serous carcinoma oligometastases. It appears that at least a small minority of these patients are curable with SBRT alone.

Second, we hypothesized that a safe broadly applicable minimal effective SBRT dose in five fractions exists for extracranial ovarian and uterine oligometastases. Our median prescription dose was 35 Gy (range, 30-50) and our local control rates at 2 and 5 years were 82 and $73 \%$. Three of the eight patients who were treated with 30 Gy in five fractions in our series failed locally; only one of 12 patients in the high-dose arm ( $\geq 35 \mathrm{~Gy}$ ) experienced local failure. Our current practice is to treat patients with a minimum dose of 35 Gy in five fractions. In our experience normal tissue dose constraints can be routinely met with this approach limiting acute and chronic toxicity. However, when safe, we dose escalate in all patients respecting published dose constraints (15). We consider dose escalation particularly important for larger tumors ( $\geq 50 \mathrm{cc}$ ) that are not resectable.

Finally, treating single ovarian and uterine oligometastases did not meaningfully increase our PFS relative to contemporaneous studies. Median PFS was just 9 months. As previously advocated by Kunos et al., in order to improve cure rates, identifying effective systemic agents to pair with SBRT is of paramount importance
(17). With the recent proliferation of novel immuno and molecular therapies there are now a large number of drugs worthy of study. Nonetheless, in patients who have been extensively treated with systemic agents and would likely continue to receive treatment for the remainder of their lives, we agree with Lazzari et al. that even a brief chemotherapy free interval may be a valuable endpoint in and of itself (18).

Limitations of this series include the fact that this is a singleinstitution, retrospective study with a relatively small patient population. Although one of the aims was to identify clinical factors that could significantly predict PFS, the limited sample size precluded this. Additionally, the relative rarity of the single metastasis presentation, diversity of primary and metastatic disease sites treated and pre- and post-SBRT systemic therapy regimens used confounds the interpretation of our outcome data. On the first point, we wished to test the hypothesis that SBRT may cure patients in the earliest stages of metastatic disease. Our long follow-up and suitable OS supports this selection criteria. However, it is possible that the presence of a single metastasis suggests a relatively indolent disease entity relative to patients with two or more metastases. Our median OS being a lengthy 4 years while our median PFS is only a mere 9 months supports this valid criticism. We concede that our results may only be applicable to patients with single metastases and not the more commonly observed oligometastatic patient with two or three metastases. Also, the diversity of tumors irradiated with varied SBRT protocols and a myriad of systemic agents used before and after treatment make it difficult to evaluate the local efficacy and clinical impact of SBRT on this patient population. Future trials, if feasible, should treat well defined not previously treated disease entities such as oligometastatic endometroid adenocarcinoma with standard SBRT and systemic therapy protocols.

\section{CONCLUSION}

The 5-year OS (46\%) and PFS (20\%) reported in this manuscript closely resemble the recently reported SABRCOMET trials significantly improved 5-year OS (42.3\%) and PFS (17.3\%) for predominately breast, lung, colorectal, and prostate oligometastatic patients treated with SBRT (20). Our results suggest that SBRT is also a safe and effective treatment for oligometastatic ovarian and uterine cancer patients. Furthermore, the long-term disease-free survivors in our cohort support the presence of a true oligometastatic state in ovarian and uterine cancer curable with SBRT alone (19). Durable local control is achievable in most metastases with a minimum dose of 35 Gy. A safe, widely applicable five fraction scheme minimizes needless toxicity in a group of patients likely to develop additional metastatic sites and ultimately die from metastatic disease. Finally, a reasonable disease-free interval (median 9 months) after such a brief local therapy (median 7 days) allows patients to quickly pursue new systemic agents if deemed necessary or recover from prior treatment (18). Further refinement of patient selection and SBRT protocols as well as the identification of suitable concurrent agents will be required to improve outcomes in the future (17). 


\section{DATA AVAILABILITY STATEMENT}

The datasets presented in this article are not readily available because: The authors are unable to share the data as we did not include data sharing provisions in the IRB (2011-210). Requests to access the datasets should be directed to collinsb@ gunet.georgetown.edu.

\section{ETHICS STATEMENT}

This retrospective study was approved by Georgetown University institutional review board (IRB-2011-210).

\section{REFERENCES}

1. Cancer Facts \& Figures. American Cancer Society (2019). Available at: https:// www.cancer.org/research/cancer-facts-statistics/all-cancer-facts-figures/ cancer-facts-figures-2019.html.

2. Chi DS, Fong Y, Venkatraman ES, Barakat RR. Hepatic resection for metastatic gynecologic carcinomas. Gynecol Oncol (1997) 66:45-51. doi: 10.1006/gyno.1997.4727

3. Bilancia R, Nardini M, Waller D. Pulmonary metastasectomy in uterine malignancy: outcomes and prognostic factors. J Thorac Dis (2017) 9:S131621. doi: $10.21037 /$ jtd.2017.04.63

4. Tree AC, Khoo VS, Eeles RA, Ahmed M, Dearnaley DP, Hawkins MA, et al. Stereotactic body radiotherapy for oligometastases. Lancet Oncol (2013) 14: e28-37. doi: 10.1016/S1470-2045(12)70510-7

5. Albuquerque KV, Singla R, Potkul RK, Smith DM, Creech S, Lo S, et al. Impact of tumor volume-directed involved field radiation therapy integrated in the management of recurrent ovarian cancer. Gynecol Oncol (2005) 96:701-4. doi: 10.1016/j.ygyno.2004.11.008

6. Rhomberg W. The Radiation Response of Sarcomas by Histologic Subtypes: A Review With Special Emphasis Given to Results Achieved With Razoxane. Sarcoma (2006) 2006. doi: 10.1155/SRCM/2006/87367

7. Fuks Z, Rizel S, Biran S. Chemotherapeutic and surgical induction of pathological complete remission and whole abdominal irradiation for consolidation does not enhance the cure of stage III ovarian carcinoma. J Clin Oncol (1988) 6:509-16. doi: 10.1200/JCO.1988.6.3.509

8. Randall ME, Filiaci VL, Muss H, Spirtos NM, Mannel RS, Fowler J, et al. Randomized phase III trial of whole-abdominal irradiation versus doxorubicin and cisplatin chemotherapy in advanced endometrial carcinoma: a Gynecologic Oncology Group Study. J Clin Oncol (2006) 24:36-44. doi: 10.1200/JCO.2004.00.7617

9. Wolfson AH, Brady MF, Rocereto T, Mannel RS, Lee Y-C, Futoran RJ, et al. A gynecologic oncology group randomized phase III trial of whole abdominal irradiation (WAI) vs. cisplatin-ifosfamide and mesna (CIM) as post-surgical therapy in stage I-IV carcinosarcoma (CS) of the uterus. Gynecol Oncol (2007) 107:177-85. doi: 10.1016/j.ygyno.2007.07.070

10. Rusthoven CG, Schefter TE. Rationale for ablation of oligometastatic disease and the role of stereotactic body radiation therapy for hepatic metastases. Hepatic Oncol (2014) 1:81-94. doi: 10.2217/hep.13.12

11. Gomez DR, Tang C, Zhang J, Blumenschein GR Jr, Hernandez M, Lee JJ, et al. Local Consolidative Therapy Vs. Maintenance Therapy or Observation for Patients With Oligometastatic Non-Small-Cell Lung Cancer: Long-Term Results of a Multi-Institutional, Phase II, Randomized Study. J Clin Oncol (2019) 37:1558-65. doi: 10.1200/JCO.19.00201

12. Scorsetti M, Comito T, Tozzi A, Navarria P, Fogliata A, Clerici E, et al. Final results of a phase II trial for stereotactic body radiation therapy for patients with inoperable liver metastases from colorectal cancer. J Cancer Res Clin Oncol (2015) 141:543-53. doi: 10.1007/s00432-014-1833-x

13. Collins BT, Erickson K, Reichner CA, Collins SP, Gagnon GJ, Dieterich S, et al. Radical stereotactic radiosurgery with real-time tumor motion tracking

\section{AUTHOR CONTRIBUTIONS}

$\mathrm{NA}$ and $\mathrm{BC}$ contributed to conception and design of the study. $\mathrm{BC}$ and $\mathrm{WB}$ contributed to data collection. NA performed data statistical analysis. All authors contributed to the article and approved the submitted version.

\section{ACKNOWLEDGMENTS}

Interim analyses of the current series were presented at the annual meetings of the American Society for Radiation Oncology (2012) and the European Society for Radiotherapy \& Oncology (2017) (21, 22).

in the treatment of small peripheral lung tumors. Radiat Oncol Lond Engl (2007) 2:39. doi: 10.1186/1748-717X-2-39

14. Degen JW, Gagnon GJ, Voyadzis J-M, McRae DA, Lunsden M, Dieterich S, et al. CyberKnife stereotactic radiosurgical treatment of spinal tumors for pain control and quality of life. J Neurosurg Spine (2005) 2:540-9. doi: 10.3171/ spi.2005.2.5.0540

15. Benedict SH, Yenice KM, Followill D, Galvin JM, Hinson W, Kavanagh B, et al. Stereotactic body radiation therapy: the report of AAPM Task Group 101. Med Phys (2010) 37:4078-101. doi: 10.1118/1.3438081

16. US Department of Health and Human Services. Common terminology criteria for adverse events (CTCAE) version 4.0. National Institutes of Health, National Cancer Institute (2009). 4(03).

17. Kunos CA, Brindle J, Waggoner S, Zanotti K, Resnick K, Fusco N, et al. Phase II Clinical Trial of Robotic Stereotactic Body Radiosurgery for Metastatic Gynecologic Malignancies. Front Oncol (2012) 2:181. doi: 10.3389/ fonc.2012.00181

18. Lazzari R, Ronchi S, Gandini S, Surgo A, Volpe S, Piperno G, et al. Stereotactic Body Radiation Therapy for Oligometastatic Ovarian Cancer: A Step Toward a Drug Holiday. Int J Radiat Oncol Biol Phys (2018) 101:650-60. doi: 10.1016/ j.ijrobp.2018.03.058

19. Hellman S, Weichselbaum RR. Oligometastases. J Clin Oncol Off J Am Soc Clin Oncol (1995) 13:8-10. doi: 10.1200/JCO.1995.13.1.8

20. Palma DA, Olson R, Harrow S, Gaede S, Louie AV, Haasbeek C, et al. Stereotactic Ablative Radiotherapy for the Comprehensive Treatment of Oligometastatic Cancers: Long-Term Results of the SABR-COMET Phase II Randomized Trial. J Clin Oncol (2020) JCO2000818. doi: 10.1200/JCO.20.00818

21. Chaudhry H, Oermann E, Suy S, Yu X, Collins SP, Barnes W, et al. Stereotactic Body Radiation Therapy (SBRT) for Single Small $(<5 \mathrm{~cm})$ Extracranial Ovarian or Uterine Cancer Metastases: Promising Local Control and Normal Tissue Tolerance With 5 Fraction Approach. Int J Radiat Oncol • Biol • Phys (2012) 84:S448. doi: 10.1016/j.ijrobp.2012.07.1187

22. Repka MC, Aghdam N, Suy S, Collins SP, Barnes W, Collins BT, et al. EP-1401: SBRT for solitary extracranial metastases from gynecologic malignancies. Radiother Oncol (2017) 123:S748-9. doi: 10.1016/S0167-8140(17)31836-4

Conflict of Interest: SC and BC serve as clinical consultants to Accuray Inc. The Department of Radiation Medicine at Georgetown University Hospital receives a grant from Accuray to support a research coordinator.

The remaining authors declare that the research was conducted in the absence of any commercial or financial relationships that could be construed as a potential conflict of interest.

Copyright (c) 2020 Aghdam, Repka, McGunigal, Pepin, Paydar, Rudra, Paudel, Pernia Marin, Suy, Collins, Barnes and Collins. This is an open-access article distributed under the terms of the Creative Commons Attribution License (CC BY). The use, distribution or reproduction in other forums is permitted, provided the original author(s) and the copyright owner(s) are credited and that the original publication in this journal is cited, in accordance with accepted academic practice. No use, distribution or reproduction is permitted which does not comply with these terms. 